\title{
Etude de l'activité chirurgicale des praticiens libéraux en Auvergne
}

\section{Study of surgical activity by liberal practitioners in Auvergne}

\author{
LAURENT DEVOIZE**, AURELIEN DURAND*, STEPHANIE TUBERT-JEANNIN ${ }^{\star \star \star}$, CHRISTOPHE DESCHAUMES**, \\ CEDRIC HUARD**, ESTELLE MACHAT-PEGON ${ }^{\star \star *}$, THIERRY ORLIAGUET ${ }^{\star \star}$, MARTINE BAUDET-POMMEL $^{* \star}$
}

\begin{abstract}
RÉSUMÉ
Les conditions pour la pratique de la chirurgie buccale sont dans une phase d'évolution : développement de l'implantologie, nécessité d'un plateau technique aux normes... Tous ces paramètres ont motivé la réalisation d'une enquête par questionnaire auprès des 830 chirurgiens dentistes auvergnats afin d'identifier les caractéristiques de la pratique chirurgicale dans cette région et de mettre en évidence les freins à cette pratique. Le taux de participation est de 41,5 \%. II apparaît que les praticiens réalisent les avulsions des dents sur l'arcade et assurent la prise en charge des patients pour les pathologies médicales courantes. De nombreux praticiens adressent leurs patients à un référent pour les actes de chirurgie buccale plus complexes et pour les patients à haut risque médical. La prise en charge de ces actes varie selon les caractéristiques des praticiens (âge, sexe, conditions d'exercice) mais ces derniers expriment quelques réticences à la pratique de la chirurgie buccale. Ils signalent des lacunes dans la formation, craignent le risque médico-légal et se plaignent de la faible rémunération de certains actes. Ces informations sont utiles pour définir d'éventuels aménagements de la formation initiale, ainsi que pour organiser la formation continue dans ce domaine. II apparaît aussi souhaitable de permettre la qualification de praticiens exclusifs en chirurgie buccale afin d'assurer la prise en charge des besoins de la population. (Med Buccale Chir Buccale 2006; 12: 63-72)
\end{abstract}

mots clés : enquête épidémiologique, chirurgie buccale

\section{SUMMARY}

Conditions of practice of buccal surgery are under way of evolution; development of implantology, necessity of technical tray in norms. All these parameters motivated the realization of an inquiry by questionnaire addressed to the 830 dental surgeons of Auvergne to identify the characteristics of surgical practice in this region and put in an obvious place the brakes for this practice. The turnout rate is $41,5 \%$. It appears that practitioners realize avulsions of teeth on arcade and assure management of patients with usual medical diseases. Numerous practitioners address their patients to a referent for acts of buccal surgery more complex and for patients with high medical risk. The management of acts of surgery varies according to the characteristics of the practitioners (age, sex, conditions of exercise). The practitioners express reservations in the practice of buccal surgery. They express lack in term of training, fear medico-legal risk and complain about the weak remuneration of certain acts. This information is useful to define possible developments of initial training, as well as to organize continuous education in this domain. It appears so desirable to allow the qualification of exclusive practitioners in oral surgery to assure management of population' needs. (Med Buccale Chir Buccale 2006; 12: 63-72)

key words : epidemiological inquiry, oral surgery médecine buccale chirurgie buccale VOL. $12, \mathrm{~N}^{\circ} 2$ 2006 page 63

* Faculté de Chirurgie Dentaire, Université Clermont 1, 63000 Clermont-Ferrand, Service d'Odontologie, CHU Clermont-Ferrand, 63000 Clermont-Ferrand

** Inserm E216, 63000 Clermont-Ferrand, Faculté de Chirurgie Dentaire, Université Clermont 1, 63000 Clermont-Ferrand, Service d'Odontologie, CHU Clermont-Ferrand, 63000 Clermont-Ferrand

*** Equipe d'accueil EA 3847, 63000 Clermont-Ferrand, Faculté de Chirurgie Dentaire, Université Clermont 1, 63000 Clermont-Ferrand, Service d'Odontologie, CHU Clermont-Ferrand, 63000 Clermont-Ferrand

Demande de tirés à part :

Laurent DEVOIZE Faculté de Chirurgie Dentaire, Université Clermont 1, 11 boulevard Charles de Gaulle, 63000 Clermont-Ferrand Accepté pour publication le 2 mai 2006 
médecine

buccale

chirurgie

buccale

VOL. $12, \mathrm{~N}^{\circ} 2$ 2006

page 64
En raison de la disparition du Certificat d'Etudes Spéciales (CES) en Stomatologie en 1991 et du succès relatif du Diplôme d'Etudes Supérieures en Stomatologie, il n'y a plus suffisamment de spécialistes pour assurer la relève des stomatologues en France. Certains estiment qu'il y aura un manque de plus de 300 spécialistes pour assurer les besoins en chirurgie de la population. De ce fait, les chirurgiens dentistes sont de plus en plus sollicités pour réaliser des actes de chirurgie buccale.

Les règles d'hygiène et d'asepsie pour la chirurgie orale et la chirurgie implantaire sont lourdes. Ces activités nécessitent un plateau technique et du personnel qualifié. Ces conditions sont difficiles à réunir dans une structure d'omnipratique libérale [1].

De plus, les compétences techniques à acquérir, le stress des actes de chirurgie buccale et leurs éventuelles complications, la faible valorisation conventionnelle de ces mêmes actes sont autant de paramètres pouvant freiner la pratique chirurgicale au sein du cabinet dentaire.

Dans ce contexte, les omnipraticiens ont donc souvent recours à des référents pour les gestes chirurgicaux. Le développement des chirurgiens dentistes référents est limité par l'absence de spécialité en chirurgie buccale en France, malgré la reconnaissance de cette spécialité dans 15 pays européens [Royaume-Uni, Allemagne, Italie, Danemark, Grèce, Irlande, Chypre, Lituanie, Hongrie, Malte, Pays-Bas, Pologne, Slovénie, Finlande et Suède (Espagne en cours de ratification)] sur 25 depuis la directive 78/686/CEE du 25 juillet 1978.

Dans un tel contexte, on peut donc s'interroger sur l'état actuel de la pratique chirurgicale, notamment en identifiant les obstacles rencontrés par les chirurgiens dentistes libéraux, leurs éventuels besoins en formation, ainsi que les modalités d'orientation des patients vers une structure spécialisée en chirurgie buccale.

C'est pourquoi une enquête a été conduite en 2005 pour évaluer dans la région Auvergne les caractéristiques de la pratique chirurgicale des chirurgiens dentistes libéraux.

\section{MATERIEL ET METHODE}

II s'agit d'une enquête régionale réalisée en 2005, basée sur un questionnaire adressé aux 830 chirurgiens dentistes libéraux de la région Auvergne. L'objectif de l'étude était d'apprécier la pratique chirurgicale dans cette région et d'évaluer les obstacles à cette activité. Dans le terme de chirurgie étaient inclus les actes d'avulsion, de chirurgie buccale, parodontale et implantaire. Le questionnaire envoyé par courrier contenait une lettre du Comité Régional de Coordination Ordinale d'Auvergne incitant les chirurgiens dentistes à répondre (enveloppe T fournie). II comprenait des questions regroupées en 6 parties :

- caractéristiques socio-démographiques du praticien (sexe, âge, université d'origine, exercice en milieu rural ou urbain, type d'activité, organisation du travail),

- conditions de la pratique chirurgicale (équipement, accès à un bloc opératoire, contact avec un référent),

- activité chirurgicale (types d'actes réalisés, types de patients pris en charge),

- formation continue en chirurgie,

- obstacles au développement de l'activité chirurgicale.

Le questionnaire a été rédigé par les chirurgiens dentistes du service d'Odontologie - département de médecine et chirurgie buccales - du CHU de Clermont-Ferrand. Après avoir été testé lors d'une phase pilote sur une dizaine de praticiens du service d'Odontologie afin d'en apprécier sa lisibilité, sa clarté et sa pertinence, le questionnaire a été modifié pour obtenir la version finale.

\section{RESULTATS}

Trois cent quarante-cinq praticiens ont répondu, ce qui correspond à un taux de réponse de $41,5 \%$. Les répondants sont âgés en moyenne de 45 ans $(45,7 \pm 10,1$ ans). Les hommes représentent $68,7 \%$ des praticiens ayant renvoyé le questionnaire complété alors qu'ils constituent $65,1 \%$ des praticiens actifs. Les praticiens ont fait leurs études dans leur grande majorité (85\%) à la Faculté de Chirurgie Dentaire de Clermont- 


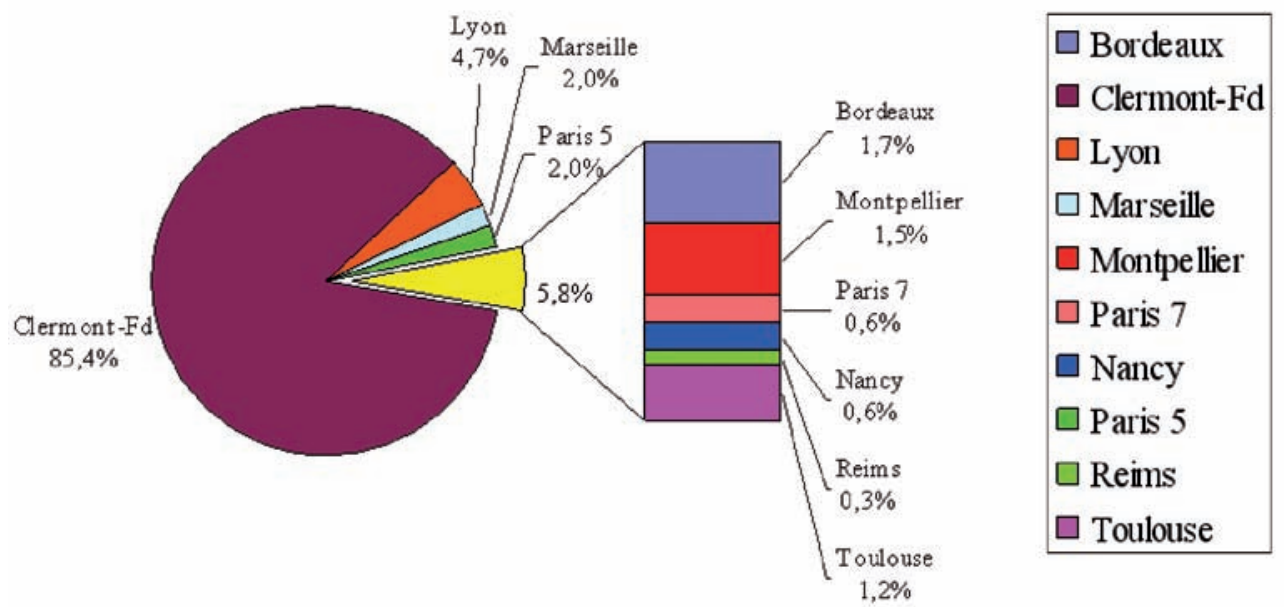

Figure 1 : Faculté d'origine des praticiens répondants, en pourcentage $(n=345)$ University where respondents have graduated, in percentage $(n=345)$

Ferrand (Fig. 1). Ces praticiens exercent avec un statut libéral $(95,6 \%)$. Ils travaillent dans des cabinets de groupe pour plus de la moitié d'entre eux $(57,4 \%)$, sans distinction de sexe. Ils se concentrent dans les zones urbaines ou péri-urbaines (68\%). Leur temps de travail moyen est de 8 demi-journées $(8,06 \pm 1,58)$, les femmes travaillant 7,5 demi-journées contre 8,4 pour leurs homologues masculins.

Le praticien travaille seul au fauteuil, sans assistance, dans $51 \%$ des cas $(62 \%$ des femmes contre $46 \%$ des hommes). Seulement $10 \%$ des praticiens disposent d'une assistante pour tous les soins. Une secrétaire est présente dans $40 \%$ des cabinets. Sur les 128 praticiens ayant accès

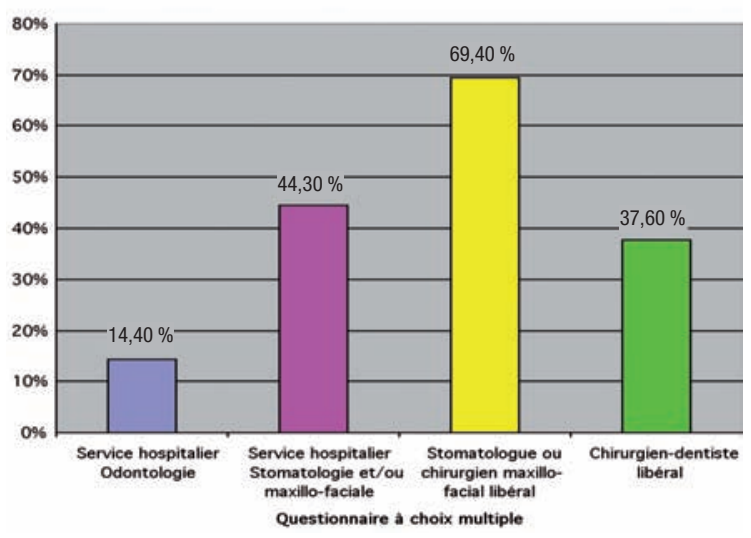

Figure 2 : Structures où sont adressés les patients pour certains actes de chirurgie $(n=327)$ Types of structures where patients are addressed for complex surgery $(n=327)$ à plusieurs fauteuils dans leur cabinet, $40 \%$ d'entre eux disposent d'un fauteuil réservé à la pratique de la chirurgie. Seuls $6,4 \%$ des praticiens font des interventions en bloc opératoire, mais $91 \%$ affirment que leurs patients ont accès à un bloc opératoire lorsque c'est nécessaire, sans préciser les conditions de cet accès.

Les 327 chirurgiens dentistes répondants qui adressent leurs patients pour certains actes de chirurgie à d'autres structures choisissent préférentiellement un confrère libéral (chirurgien dentiste ou médecin) et plus rarement un service d'Odontologie (14,4 \%) (Fig. 2). Il apparaît que $9 \%$ des praticiens adressent systématiquement leurs patients à des confrères privés, même pour les actes les plus simples.

Si tous les praticiens réalisent les avulsions simples, ils ne sont que $51 \%$ à réaliser les autres actes de chirurgie buccale, $37 \%$ de la chirurgie parodontale, et $18 \%$ de la chirurgie implantaire. Le détail des actes réalisés en pratique courante est présenté dans le Tab. 1. Soixante dix-sept pour cent des praticiens interrogés prennent en charge de façon régulière des patients ayant des antécédents médicaux qui nécessitent des soins particuliers. Parmi les pathologies concernées, la prise en charge des patients ayant des antécédents d'irradiation cervico-faciale, en cours de chimiothérapie, greffés rénaux ou cardiaques semble poser problème puisque moins de la moitié des chirurgiens dentistes acceptent de les gérer (Tab. 2). médecine

buccale chirurgie buccale

VOL. $12, \mathrm{~N}^{\circ} 2$ 2006

page 65 
médecine

buccale

chirurgie

buccale

VOL. $12, \mathrm{~N}^{\circ} 2$ 2006

page 66
Tableau 1 : Actes de chirurgie réalisés en pratique courante $(n=345)$ Type of dental surgery done in current practice $(n=345)$

\begin{tabular}{|l|l|}
\hline Actes & Nombre (Pourcentage) \\
\hline Avulsions simples & $\mathbf{3 4 4}(99,7 \%)$ \\
\hline Avulsion des dents de sagesse sur l'arcade & $\mathbf{2 8 8}(83,5 \%)$ \\
\hline Avulsion avec alvéolectomie & $\mathbf{2 6 6}(77,1 \%)$ \\
\hline Elongation coronaire & $\mathbf{1 8 3}(53,0 \%)$ \\
\hline Freinectomie & $\mathbf{1 4 9}(43,2 \%)$ \\
\hline Résection apicale & $\mathbf{1 4 1}(40,9 \%)$ \\
\hline Avulsion des dents de sagesse incluses & $\mathbf{1 3 5}(39,1 \%)$ \\
\hline Chirurgie parodontale & $\mathbf{1 0 6}(30,7 \%)$ \\
\hline Chirurgie préprothétique (muqueuse et osseuse) & $\mathbf{9 8}(28,4 \%)$ \\
\hline Avulsion des autres dents incluses & $\mathbf{7 7}(22,3 \%)$ \\
\hline Avulsion des dents canines incluses & $\mathbf{7 4}(21,4 \%)$ \\
\hline Chirurgie orthodontique (désinclusion canine) & $\mathbf{7 2}(20,9 \%)$ \\
\hline Pose d'implant & $\mathbf{6 4}(18,6 \%)$ \\
\hline Exérèse de lésions muqueuses & $\mathbf{5 9}(17,1 \%)$ \\
\hline Biopsie & $\mathbf{3 7}(10,7 \%)$ \\
\hline
\end{tabular}

Tableau 2 : Prise en charge des patients à risque médical par les praticiens $(n=331)$ Management of patients with a medical risk by the practitioners $(n=331)$

\begin{tabular}{|l|l|}
\hline Pathologies & Nombre (Pourcentage) \\
\hline Diabétique & $\mathbf{3 1 6}(95,5 \%)$ \\
\hline Sous anti-coagulants & $\mathbf{2 9 6}(89,4 \%)$ \\
\hline Hypertendu & $\mathbf{2 9 3}(88,5 \%)$ \\
\hline Avec antécédents d'infarctus du myocarde & $\mathbf{2 8 0}(84,6 \%)$ \\
\hline Poly-médicamenté & $\mathbf{2 7 4}(82,8 \%)$ \\
\hline A risque d'endocardite & $\mathbf{2 7 2}(82,2 \%)$ \\
\hline Avec un problème infectieux (SIDA, hépatite B ou C) & $\mathbf{2 3 1}(69,8 \%)$ \\
\hline Cancéreux & $\mathbf{2 3 0}(69,5 \%)$ \\
\hline Insuffisant cardiaque & $\mathbf{2 2 5}(68,0 \%)$ \\
\hline Greffés (rénal ou cardiaque) & $\mathbf{1 6 5}(49,9 \%)$ \\
\hline Cancéreux en cours de chimiothérapie & $\mathbf{1 4 5}(43,8 \%)$ \\
\hline Cancéreux avec antécédents d'irradiation buccale & $\mathbf{1 3 6}(41,1 \%)$ \\
\hline
\end{tabular}

Ces 5 dernières années, $47 \%$ des praticiens ont participé à une formation en médecine buccale et chirurgie buccale. Seuls 61 praticiens ont un ou plusieurs diplôme(s) ou attestations(s) complémentaire(s) dans le domaine de la chirurgie buccale ; $30 \%$ ont obtenu un CES, $25 \%$ un 


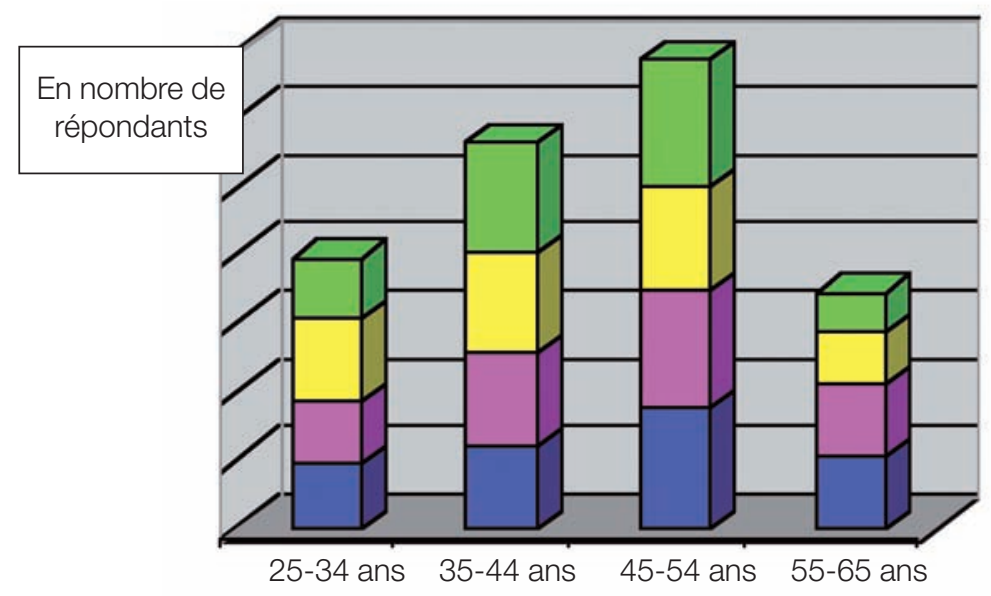

- Chirurgie implantaire

- Chirurgie parodontale

- Autres actes de chirurgie

- Avulsions simples

Figure 3 : Types de pratique chirurgicale et âge des praticiens Types of surgical practice and age of practitioners

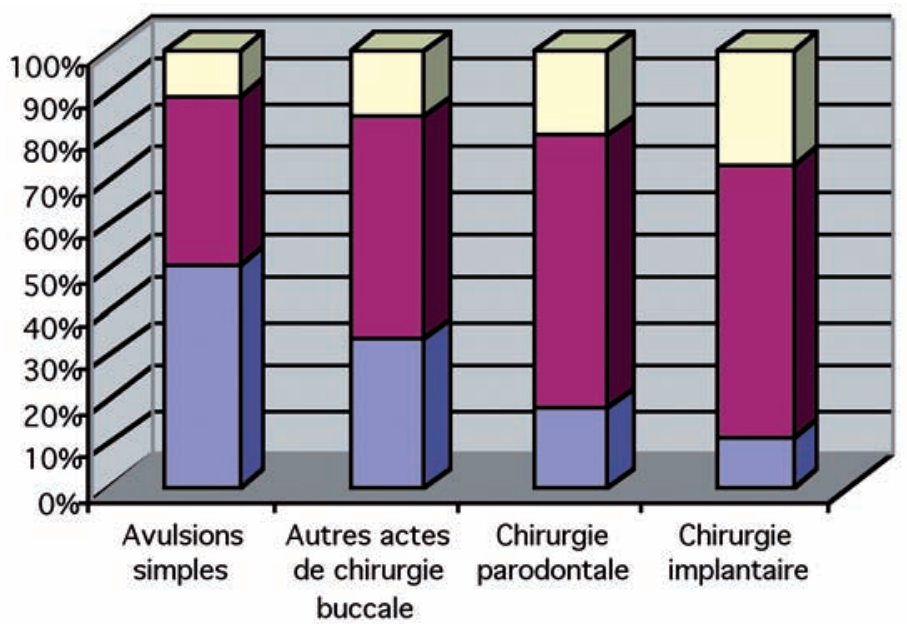

口 Quatre mains

- Variable

口 Deux mains médecine

buccale

chirurgie

buccale

VOL. $12, \mathrm{~N}^{\circ} 2$

2006

page 67

Figure 4 : Types de pratique chirurgicale et organisation du travail

Types of surgical practice and practice conditions

Nous avons recherché le lien existant entre la pratique chirurgicale et les caractéristiques sociodémographiques des praticiens. Si tous les répondants réalisent des avulsions simples, $61,6 \%$ des hommes et seulement $29 \%$ des femmes effectuent d'autres actes de chirurgie. La différence s'accentue pour la pratique de la parodontologie chirurgicale puisque seulement $13 \%$ des femmes ont cette activité contre $48 \%$ des hommes. Sur les 61 répondants réalisant des actes de chirurgie implantaire, il n'y a qu'une seule femme. Les répondants ont été regroupés en quatre tranches d'âge : 25-34 ans, 35-44 ans, 4554 ans, 55-65 ans. La chirurgie implantaire est essentiellement réalisée par les moins de 55 ans, puisque $17 \%$ des $25-34$ ans, $19 \%$ des $35-44$ ans et $18 \%$ des $45-54$ ans pratiquent ce type de chirurgie contre moins de $10 \%$ pour les plus de 55 ans (Fig. 3). Une corrélation semble s'établir entre le coefficient technique des actes chirurgicaux et le suivi d'une formation continue dans ce domaine. En effet, moins de la moitié des répondants effectuant des avulsions simples a suivi une formation, alors que plus de $73 \%$ des praticiens pratiquant la chirurgie parodontale et plus de $95 \%$ des praticiens pratiquant la chirurgie implantaire ont effectué au moins une séance de formation continue lors des cinq dernières années. 
Les actes de chirurgie orale, autres que les avulsions simples, sont plus volontiers réalisés par des praticiens ayant une assistance au fauteuil (Fig. 4). La pratique chirurgicale implantaire est moins courante pour les répondants exerçant en milieu rural $(11,3 \%)$ que pour ceux exerçant en milieu urbain $(19,5 \%)$ ou périurbain $(25 \%)$. II apparaît que $38 \%$ des praticiens sont réticents à pratiquer les actes de chirurgie buccale. Les raisons évoquées pour expliquer cette réticence sont présentées dans la Fig. 5 . Les 5 motifs le plus souvent évoqués sont le manque de formation chirurgicale et médicale, le stress occasionné par ce type d'acte, le risque médico-légal et la faible rémunération de certains actes chirurgicaux dans la nomenclature. La réticence à pratiquer la chirurgie orale varie selon le profil du praticien. Les femmes sont $60 \%$ à exprimer leurs réticences (12\% sont franchement réticentes) contre $28 \%$ des hommes (4,6\% sont franchement réticents). Environ $42 \%$ des praticiens entre $45-54$ ans et $44 \%$ des praticiens entre $55-65$ ans sont réticents à l'égard de la pratique chirurgicale, alors qu'ils sont moins d'un tiers entre 25-34 ans.

Moins de $25 \%$ des praticiens ayant participé, au moins à une séance de formation continue lors des cinq dernières années, sont réticents pour pratiquer la chirurgie buccale, contre plus de 50\% des praticiens qui n'ont pas suivi une telle formation (Fig. 6). Plus de $52 \%$ des praticiens exerçant seul au fauteuil, contre $25 \%$ bénéficiant d'une assistance partielle et moins de $15 \%$ travaillant à quatre mains, sont réticents à la pratique de la chirurgie buccale (Fig. 7). médecine

buccale

chirurgie

buccale

VOL. $12, \mathrm{~N}^{\circ} 2$ 2006

page 68
Manque de formation chirurgicale

Stress

Manque de formation médicale

Risque médico-légal

Rapport prise de risque/rénumération de l'acte défavorable

Rémunération insuffisante de ce type d'acte

Absence d'une assistance qualifiée

Manque d'équipement

Problème de mise aux normes des locaux

Coût de la stérilisation, problème d'asepsie

Refus de vos patients à accepter cet acte en dehors d'une structure hospitalière

Activité à temps partie compromettant le suivi des patients

Statut provisoire/remplaçant ou collaborateur
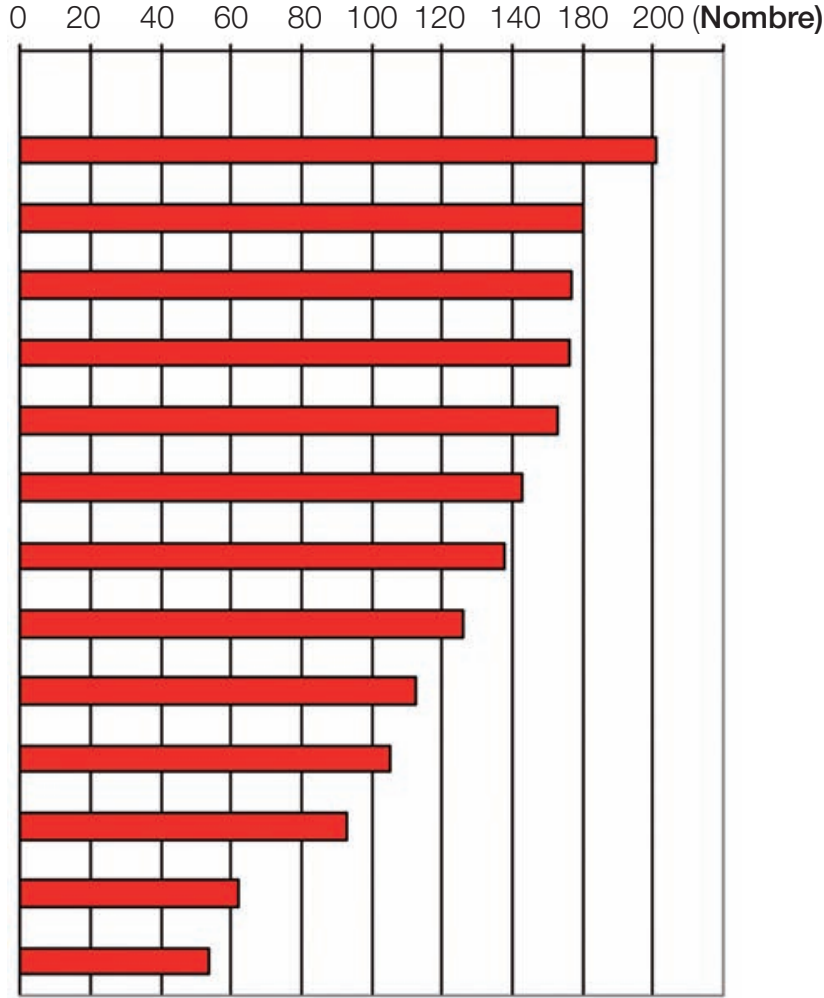

Figure 5 : Motifs de la réticence à pratiquer la chirurgie buccale $(n=224)$

Reasons of the reluctance to practice surgery $(n=224)$ 


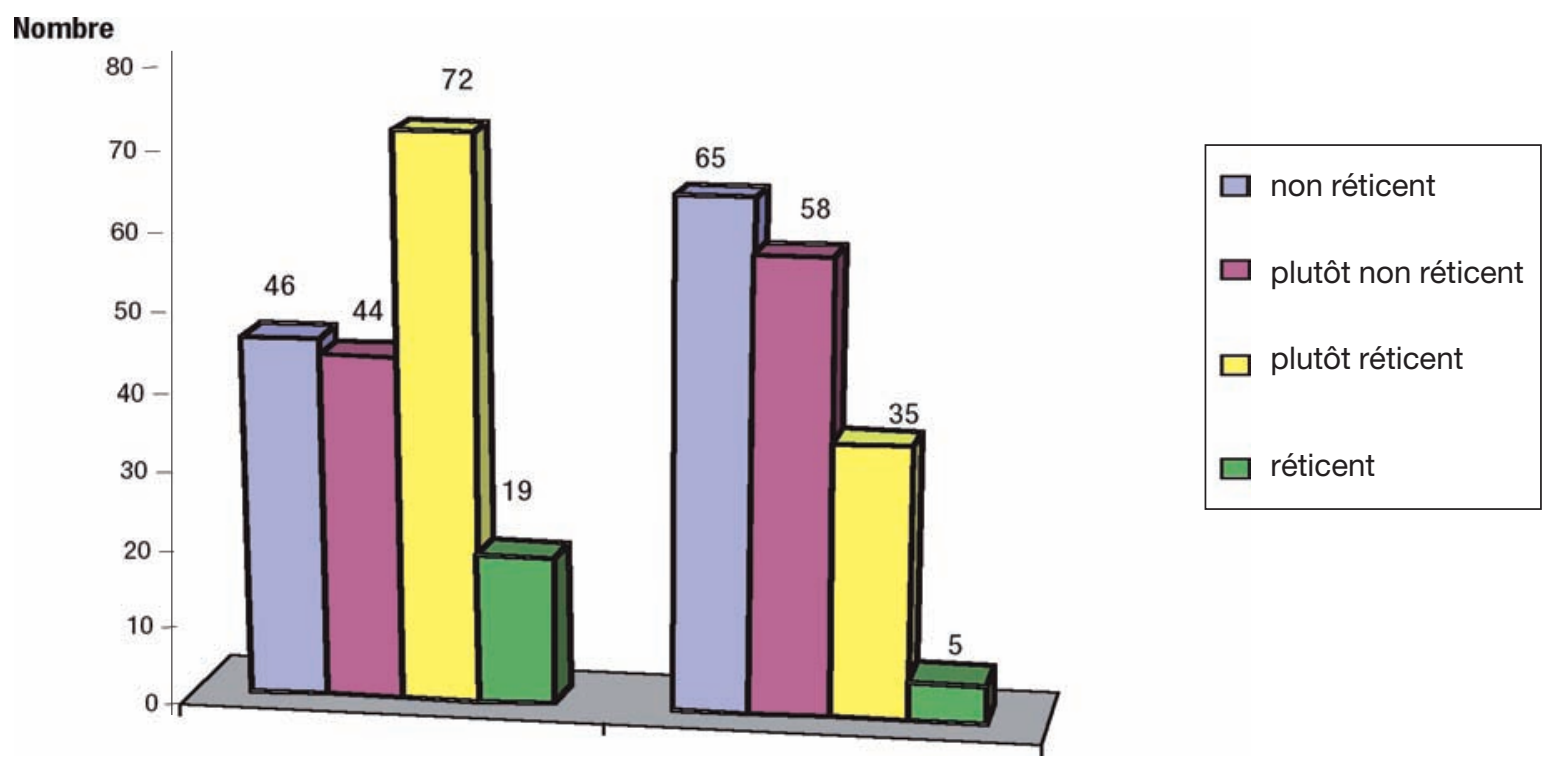

Figure 6 : Réticence à la pratique d'actes de chirurgie et formation continue $(n=344)$ Reluctance to practice surgery and adult continuous education $(n=344)$

médecine

buccale chirurgie buccale

VOL. $12, \mathrm{~N}^{\circ} 2$ 2006

page 69

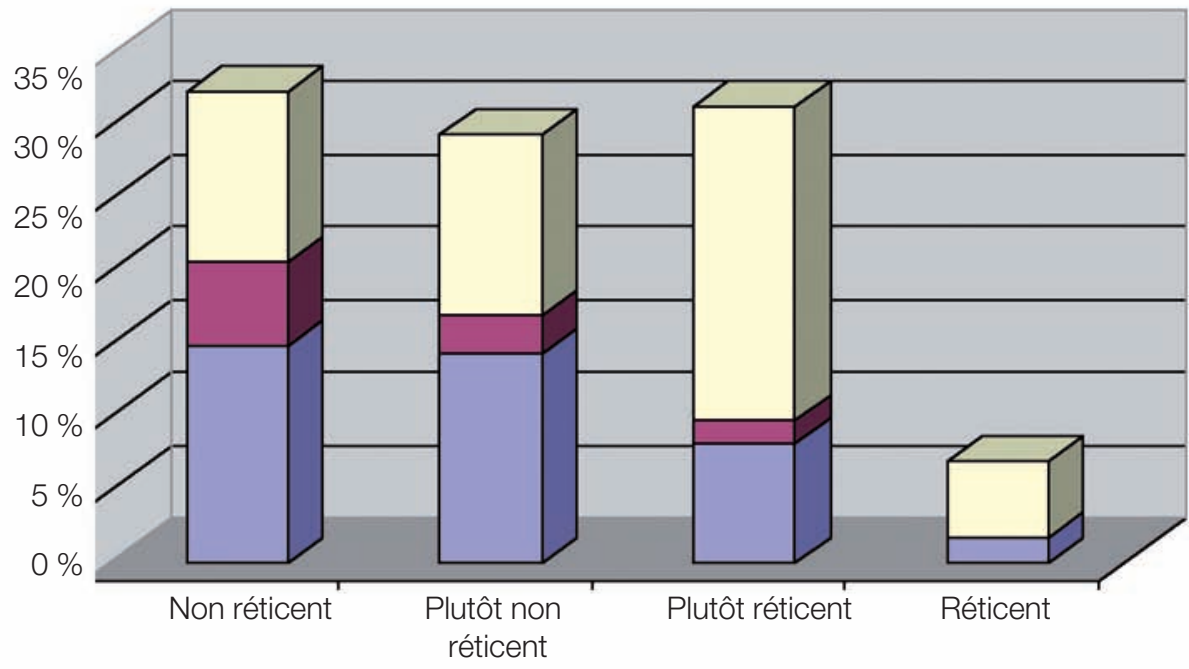

ㅁ 2 mains

4 mains

$\square$ Variable

Figure 7 : Réticence à la pratique chirurgicale et conditions d'exercice $(n=343)$ Reluctance to practice surgery and practice conditions $(n=343)$ 


\section{DISCUSSION}

Le questionnaire a été envoyé début 2005 aux adresses professionnelles de 830 praticiens de la région Auvergne, soit plus de $90 \%$ des praticiens inscrits au Tableau de l'Ordre des chirurgiens dentistes. Le taux de réponse à cette enquête a été de $41,5 \%$, chiffre acceptable si l'on considère que la participation des chirurgiens dentistes et des médecins français à des questionnaires postaux oscille entre $24 \%$ et $56,5 \%$ [2]. Cette étude a permis de décrire l'état de la pratique de chirurgie buccale en Auvergne. II apparaît que les praticiens réalisent les avulsions des dents présentes sur l'arcade. Ils assurent la prise en charge des patients pour les pathologies médicales courantes. En revanche, il semble que de nombreux praticiens adressent leurs patients à un praticien référent pour les actes de chirurgie médecine buccale chirurgie buccale

VOL. $12, \mathrm{~N}^{\circ} 2$ 2006

page 70 orale plus complexes et pour les patients ayant un risque médical élevé. La prise en charge des actes de chirurgie buccale varie selon les caractéristiques des praticiens. En particulier, les femmes semblent plus fréquemment adresser leurs patients à un praticien référent. Ceci peut s'expliquer par le fait que les femmes auraient un temps de présence au fauteuil plus faible et donc un volume d'activité inférieur ${ }^{[3,4]}$. L'activité féminine ne favorise pas le recrutement d'une assistance au fauteuil permettant d'avoir les conditions optimales pour la pratique chirurgicale. Les bilans d'activité fournis par la Caisse Nationale d'Assurance Maladie montrent que l'activité moyenne des femmes est inférieure d'environ $30 \%$ à celle des hommes sur les années 1999 à 2002 [5]. La différence pour l'activité chirurgicale étant de plus de $36 \%$ pour l'année 2002 : les résultats de notre enquête confirment ces données. Les femmes auraient aussi une visée plus conservatrice que les praticiens masculins et tendance à délaisser l'activité chirurgicale pour privilégier la réalisation de soins conservateurs ${ }^{[5,6]}$. Il faut aussi souligner les obligations inhérentes à la chirurgie, comme les contraintes liées à la gestion de complications postopératoires, semblent susciter la réticence chez certains praticiens, en particulier féminins [7]. On peut aussi se demander s'il n'existe pas, comme aux Etats-Unis, des préjugés sur l'activité chirurgicale qui reste aux yeux des patients américains une activité plutôt masculine, rendant la spécialisation peu attractive pour les femmes ${ }^{[8]}$. La pratique de la chirurgie parodontale et implantaire est plus répandue chez les 25-34 ans que chez les 55-65 ans. L'implantologie étant une discipline récente, il est possible que le groupe des 25-34 ans ait bénéficié d'une formation initiale sur le sujet contrairement aux plus de 55 ans. La participation a des séances de formation continue sur la chirurgie buccale varie aussi selon l'âge, la tranche d'âge des 35-44 ans étant la plus susceptible de s'être formée. Les jeunes (25-34 ans) n'éprouvent peutêtre pas encore le besoin de formation estimant leur formation initiale suffisante. De même, les plus de 55 ans, plus proches de la retraite n'ont peut-être pas envie de se lancer dans une nouvelle activité, en particulier de type chirurgical. L'étude montre que certains chirurgiens dentistes sont réticents pour pratiquer les actes de chirurgie buccale. Les motifs les plus fréquemment invoqués sont le manque de formation, le stress occasionné par ce type d'acte, la peur du risque médico-légal et la faible rémunération des actes chirurgicaux dans la nomenclature. La réticence à pratiquer la chirurgie varie selon le profil du praticien. Il est important de rappeler qu'il s'agit d'une réticence et non d'une absence de pratique. Les praticiens nous informent sur ce qui les freinent, voire les arrêtent pour certains, dans leur activité. Le manque de formation et le stress sont des facteurs intimement liés ${ }^{[1,9]}$. La formation initiale générale est probablement insuffisante pour la pratique de la chirurgie buccale complexe. A cela s'ajoute le fait que les praticiens participent peu à la formation continue en chirurgie avec une préférence pour la chirurgie parodontale et implantaire. Ils semblent attirés par les orientations récentes et montrent une désaffection pour les domaines plus classiques comme la chirurgie buccale [2]. II serait donc souhaitable pour réduire ces lacunes dans la formation que le décret d'application de la loi du 11 août 2004, instituant la Formation Continue obligatoire, soit publié rapidement. Le caractère coercitif de cette mesure devrait orienter un plus grand nombre de praticiens vers une formation en chirurgie et, donc comme le laissent percevoir 
les résultats de l'enquête, permettre une meilleure prise en charge chirurgicale. II faudrait aussi que la qualification en chirurgie buccale existant dans 15 des 25 Etats de l'Union Européenne soit adoptée en France, afin de permettre à des praticiens chirurgiens dentistes de l'exercer en exclusivité. Cette création donnerait la possibilité aux confrères d'adresser leurs patients à un chirurgien dentiste référent dans de bonnes conditions. L'absence actuelle de spécialisation crée un flou auprès des patients et des praticiens sur le niveau réel de qualification. Cette situation est donc dommageable au développement des réseaux dans le domaine de la chirurgie buccale.

Le risque médico-légal est beaucoup plus médiatisé depuis quelques années et les patients manifestent une exigence croissante dans le domaine de la santé. Pourtant, les données du Groupe des Assurances Mutuelles Médicales (GAMM) qui concerne près de 12000 chirurgiens dentistes (28\% des praticiens), recensent 437 déclarations de litiges en 2003 contre 499 en 2001. Sur l'ensemble de ces déclarations, peu étaient en rapport avec la pratique chirurgicale : 50 en 2001 et 41 en 2003. La sinistralité (qui représente le rapport du nombre de cas de contentieux sur le nombre d'assurés) de l'activité chirurgicale était donc de 0,41\% en 2001 et de 0,33\% en 2003 [10] alors que l'activité chirurgicale des praticiens augmente régulièrement en Auvergne ${ }^{[11]}$. Ces résultats sont donc en décalage avec l'à priori qui veut que le risque lié à la pratique de la chirurgie soit croissant. La peur du chirurgien dentiste de connaître un contentieux ne semble donc pas justifiée.

Les actes chirurgicaux sont peu rentables, voire déficitaires. Leur tarification conventionnelle est

\section{RÉFÉRENCES}

1 - BOUET JF. Exercice exclusif de la chirurgie buccale et Diplôme d'Etudes Supérieures en Chirurgie Buccale, étude et prospective en 1995. Thèse Chirurgie Dentaire, Montpellier 1, 1995 ; n 15-81-11-03 ; 18-9.

2 - BERAUD C. Le point sur la formation permanente des chirurgiens dentistes du Puy-de-Dôme en odontologie en effet sous-évaluée. La lettre clé DC n'a augmenté entre 1980 et 2005 que de $14 \%$, contrairement à la tarification de la consultation qui a connu une augmentation de $227 \%$ pendant cette même période ${ }^{[12]}$. Cette stabilité des tarifs des actes chirurgicaux dans la NGAP pose problème dans la mesure où les charges des cabinets dentaires ne cessent de croître et représentent maintenant près de $60 \%$ du chiffre d'affaire ${ }^{[13]}$. La réticence et le désintérêt croissants des praticiens pour l'activité de chirurgie buccale est donc compréhensible ${ }^{[9]}$. Les praticiens, dans une logique de gestionnaire, peuvent préférer éviter ces actes et référer les patients à des praticiens spécialisés en chirurgie.

\section{CONCLUSION}

Cette enquête a permis de recueillir des données sur l'état de la pratique chirurgicale en Auvergne en 2005. Les praticiens ont exprimé de nombreuses réticences pour la pratique de la chirurgie buccale. Ils ont signalé les lacunes existant dans leur formation. Ils ont une perception excessive du risque médico-légal et se plaignent de la faible rémunération de certains actes. Tout ceci concourt à augmenter le niveau de stress lors de la pratique des actes de chirurgie orale et tend à réduire la capacité de prise en charge des actes en chirurgie. Les informations recueillies pourraient constituer une base pour proposer des aménagements dans la formation initiale, ainsi que pour organiser la formation continue dans ce domaine. Il apparaît aussi souhaitable de permettre dans les meilleurs délais la qualification de praticiens exclusifs en chirurgie buccale. \begin{tabular}{l}
$\begin{array}{l}\text { médecine } \\
\text { buccale } \\
\text { chirurgie } \\
\text { buccale }\end{array}$ \\
\hline voL. $12, N^{\circ} 2$ \\
2006 \\
\hline page 71
\end{tabular} préventive et restauratrice. Thèse Chirurgie Dentaire, Clermont-Ferrand 1, 2004 ; n 11-04.

3 - Bois D, MassonnaUd J, Melet J. Mission odontologique. 2003, 9.

4 - MuRRAY JJ. Better opportunities for women dentists: a review of the contribution of women dentists to the workforce, 3rd. Dent J ; 2002. 
5 - PeRCHOUx L. La féminisation de la profession va-t-elle se traduire par une modification d'activité ? Thèse Chirurgie Dentaire, Clermont-Ferrand 1, 2004 ; n²004CLF1D019.

6 - DomeJeAn-Orliaguet S, TuBeRT-JeAnNin S, RIORDAN PJ, ESPELID I. French dentist's restorative treatment decisions. Oral Health Prevent Dent 2004 ; 2: 125-31.

7 - Romestaing P. Femme : une chance pour la médecine générale ? Panorama Med. 2004 ; 4939 : 20-4.

8 - RISSER MJ, LASKIN DM. Women in oral and maxillofacial surgery: factors affecting career choices, attitudes, and practice characteristic. J Oral Maxillofac Surg 1996 ; 54: 753-7.

9 - VILLEMEY D. Limites de l'exercice de la chirurgie au cabinet dentaire. Thèse Chirurgie Dentaire, Lyon 1, 1985 ; n०42-12-85-54.
10 - Responsabilité. Le risque des professions de santé en 2003. Revue de formation sur le risque médical 2003. GAMM 2005 ; hors série mars 2005 : 21-2.

11 - Rapports d'activité de la Caisse Régionale d'Assurance Maladie de la région Auvergne. Section Statistique 2002 et 2003.

12 - Centre Odontologique de Médicométrie et d'Evaluations. Carnet statistique. 1997 ; 96.

13 - Rapport Yahiel. Inspection Générale des Affaires Sociales (IGAS) ; 2001 (disponible sur le site www.social.gouv.fr/htm/actu/mal_prof/sommaire). médecine

buccale

chirurgie

buccale

VOL. $12, \mathrm{~N}^{\circ} 2$ 2006

page 72 